\title{
Electrical transport and low-temperature scanning tunneling microscopy of microsoldered graphene
}

V. Geringer ${ }^{1,3}$, D. Subramaniam ${ }^{1,3}$, A. K. Michel ${ }^{1,3}$, B. Szafranek ${ }^{2}$, D. Schall ${ }^{2}$, A. Georgi ${ }^{1,3}$, T. Mashoff ${ }^{1,3}$, D. Neumaier ${ }^{2}$, M. Liebmann ${ }^{1,3}$ and M. Morgenstern ${ }^{1,3}$

${ }^{1}$ II. Institute of Physics, RWTH Aachen University, Otto-Blumenthal-Straße, 52074 Aachen

${ }^{2}$ Advanced Microelectronic Center Aachen (AMICA), AMO GmbH, Otto-Blumenthal-Straße 25, 52074 Aachen, ${ }^{3}$ JARA: Fundamentals of Future Information Technology

(Dated: 9 March 2022)

Using the recently developed technique of microsoldering, we perform systematic transport studies of the influence of PMMA on graphene revealing a doping effect of up to $\Delta \mathrm{n}=3.8 \times 10^{12} \mathrm{~cm}^{-2}$, but negligible influence on mobility and hysteresis. Moreover, we show that microsoldered graphene is free of contamination and exhibits very similar intrinsic rippling as found for lithographically contacted flakes. Finally, we demonstrate a current induced closing of the previously found phonon gap appearing in scanning tunneling spectroscopy, strongly non-linear features at higher bias probably caused by vibrations of the flake and a B-field induced double peak attributed to the 0.Landau level. 
The discovery of graphene in $2004^{112}$ with its exceptional room-temperature mobility and its unconventional Quantum Hall effect boosted a wealth of theoretical ${ }^{3}$ and experimental ${ }^{4}$ work. However, several basic properties like the morphology of the flakes ${ }^{516}$ or the limiting

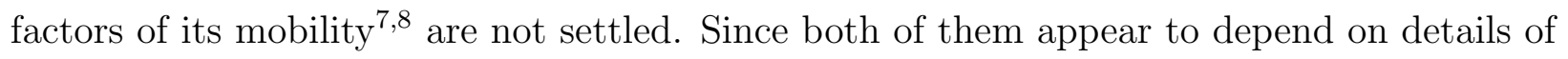
the preparation process, it is crucial to investigate well defined samples. Here, we use the recently developed technique of microsoldering 9910 in order to avoid the dirt usually induced by lithography. Indeed, in contrast to lithographically contacted samples, the microsoldered graphene is free of contamination as evidenced by scanning tunneling microscopy (STM). Thus, we could probe the influence of PMMA revealing that PMMA leads to a considerable $n$-doping up to $3.8 \times 10^{12} \mathrm{~cm}^{-2}$, while mobility and voltage induced hysteresis are barely changed. Using the clean sample, which exhibits intrinsic corrugation ${ }^{6}$, we investigated the local spectroscopic properties of the flake at $T=5 \mathrm{~K}$. We show that the phonon-induced gap found recently by Zhang et al ${ }^{11}$ is closed at higher tunneling current probably due to a local heating of the sample. Additional features appear in scanning tunneling spectroscopy (STS) at higher bias. They are not related to the local density of states (LDOS), but are most likely induced by strongly non-linear vibrations. Finally we observe a B-field induced feature, which we attribute to the 0.Landau level.

Fig. 1(a) and (b) show optical images of two microsoldered monolayers of graphene, which are prepared under ambient conditions by mechanical exfoliation on a $90 \mathrm{~nm} \mathrm{SiO}_{2}$ layer on Si(001), identified by Raman spectroscopy and soldered by drawing liquid indium on top of the flake using a micromanipulator ${ }^{9}$. The majority of the resulting contacts exhibits Ohmic behavior with contact resistances of $1-50 \mathrm{k} \Omega$. Fig. 1(c) and (d) show STM images of a graphene flake contacted by standard electron beam lithography and lift-off ${ }^{6}$ (c) and by microsoldering $(\mathrm{d})$. They are recorded at $T=5 \mathrm{~K}$ in ultra-high vacuum and are slightly high-pass filtered in order to suppress the rippling, thereby increasing the visibility of the atomic resolution. The lithographically contacted flake exhibits clusters of dirt with heights up to $2 \mathrm{~nm}$ which indicate the remaining resist. In contrast, the microsoldered sample is free of contamination. We found that about $10 \%$ of the flake regions far away from the contacts and $30 \%$ of the regions close to the contact are covered with clusters of dirt after lithography, but we never found such contamination on microsoldered samples imaging several $\mu \mathrm{m}^{2}$ in each case. The inset of Fig. 1(e) shows a larger scale image of the microsoldered graphene exhibiting an intrinsic rippling of amplitude $A=1 \mathrm{~nm}$ very similar to the rippling found 

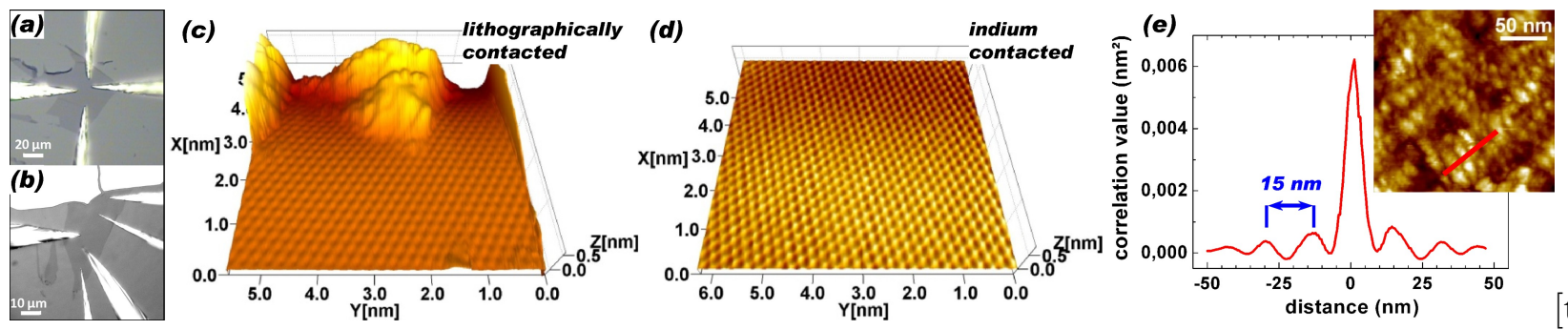

$[\mathrm{tp}]$

FIG. 1. (Color online) (a), (b) Optical images of microsoldered graphene flakes with four (a) and five (b) indium contacts. (c) STM image of a lithographically contacted graphene monolayer on $\mathrm{Si} / \mathrm{SiO}_{2}\left(\mathrm{~T}=5 \mathrm{~K}\right.$, tunneling current $\mathrm{I}_{T}=0.2 \mathrm{nA}$, tunneling voltage $\left.\mathrm{U}_{T}=1 \mathrm{~V}\right)$; remaining dirt (probably PMMA) is visible in the top part of the image. (d) STM image of a microsoldered monolayer of graphene on $\mathrm{Si} / \mathrm{SiO}_{2}\left(\mathrm{~T}=5 \mathrm{~K}, \mathrm{I}_{T}=0.5 \mathrm{nA}, \mathrm{U}_{T}=0.5 \mathrm{~V}\right)$. (e) Inset: large-scale STM image of microsoldered graphene $\left(\mathrm{T}=5 \mathrm{~K}, \mathrm{I}_{T}=1 \mathrm{nA}, \mathrm{U}_{T}=0.5 \mathrm{~V}\right)$; main: correlation function averaged along the direction indicated as a solid line in the inset; dominating wavelength is marked.

on lithographically contacted samples ${ }^{6 / 12}$. The correlation function (main image) is taken along the main direction of rippling (line in inset) being $30^{\circ}$ with respect to the $\mathrm{C}-\mathrm{C}$ bond direction. The rippling again shows a preferential wavelength of $15 \mathrm{~nm}$ evidencing that intrinsic rippling does not depend on the contact procedure.

The gate voltage dependent 4-point resistance $(\mathrm{T}=295 \mathrm{~K})$ of a microsoldered sample is shown in Fig. 2(a). The black curve measured directly after microsoldering shows a hysteresis $\Delta V_{\text {Gate }}=4 \mathrm{~V}$ and a Dirac point at $\mathrm{V}_{\text {Dirac }}=9 \mathrm{~V} / 13 \mathrm{~V}$ depending on sweep direction. Using five different samples, we get a mobility of $\mu=3200 \pm 600 \mathrm{~cm}^{2} / \mathrm{Vs}$ from van-derPauw measurements in agreement with two-point measurements of ${ }^{9}$. In vacuum $(\mathrm{p}=2 \times$ $10^{-5}$ mbar), $\mu$ increases by $50 \%$ after $18 \mathrm{~h}$ at $\mathrm{T}=50^{\circ} \mathrm{C}$. The hysteresis $\Delta V_{\text {Gate }}$ strongly depends on gate voltage range, sweep rate and surrounding atmosphere. For the sake of comparison, all curves in Fig. 2 are measured with very similar parameters as used for lithographically contacted samples in 13 . In ambient conditions, we find $V_{\text {Dirac }}=3-9 \mathrm{~V}$ during downward sweep and $\Delta V_{\text {Gate }}=3-4 \mathrm{~V}$. While $\Delta V_{\text {Gate }}$ is very similar to the results of 13 (after correcting for different $\mathrm{SiO}_{2}$ thickness), $V_{\text {Dirac }}$ shows much less scatter. By changing the environment to pure nitrogen and vacuum, $V_{\text {Dirac }}$ and $\Delta V_{\text {Gate }}$ are continuously reduced down to $V_{\text {Dirac }}=2 \mathrm{~V}$ and $\Delta V_{\text {Gate }}=0.5 \mathrm{~V}$ (Fig. 2(b)). This improvement is well known also for lithographically contacted samples and usually attributed to the removing of water and 

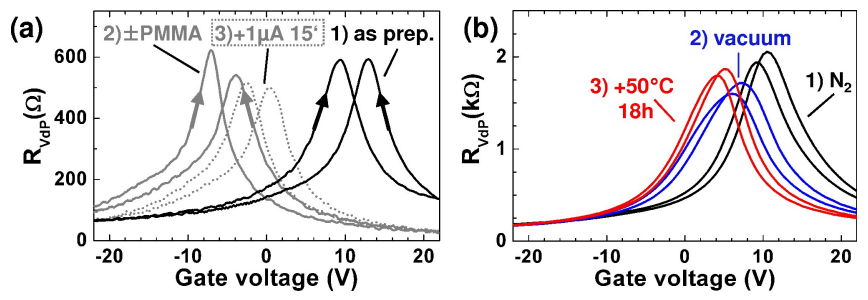

FIG. 2. (Color online) (a) Four-terminal van der Pauw measurement of the microsoldered graphene monolayer shown in Fig. 1(a) (ambient conditions); sweep rate: $0.5 \mathrm{~V} / \mathrm{s}$; black curve: as-prepared sample, grey curve: same sample after coverage with PMMA and subsequent cleaning, dotted grey curve: same sample after additional current-induced heating by $1 \mu \mathrm{A}$; measurement direction is marked by arrows. (b) Four-terminal resistance of microsoldered graphene in nitrogen (right curves), vacuum (middle curves) and after additional vacuum annealing at $50^{\circ} \mathrm{C}$ for $18 \mathrm{~h}$ (left curves).

an according reduction of charge trapping 15 .

Since the main difference between microsoldered and lithographically contacted samples is the scatter of $V_{\text {Dirac }}$, we investigate the influence of PMMA, probably the major contamination after lithography, in more detail. The three curves in Fig. 2(a) show the same sample directly after microsoldering (black), after covering the surface with PMMA using a solution in anisol and a subsequent standard cleaning procedure of rinsing the sample in acetone and propanol (grey full line), and after additional heating by currents of $1 \mu \mathrm{A}$ for several minutes (dotted grey line) ${ }^{16}$. The curves recorded after PMMA contamination but without cleaning look very similar to the ones with cleaning. Obviously neither $\Delta V_{\text {Gate }}$ nor the mobility (steepness of curves) are strongly influenced by PMMA, but a significant n-type doping results, which is straightforwardly deduced to be $\Delta \mathrm{n}=3.8 \times 10^{12} \mathrm{~cm}^{-2}$. Moreover, the resistance curve is more asymmetric after contamination and, thus, comparable to most

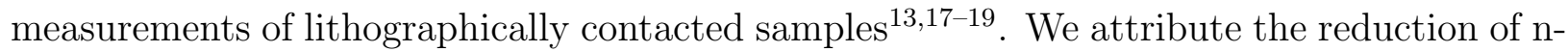
doping by current heating (dotted curve) to removing of solvents. Further heating by $10 \mu \mathrm{A}$ does not shift $V_{\text {Dirac }}$ anymore and $V_{\text {Dirac }}$ can be shifted reversibly between the two points (grey curves) by repeated PMMA contamination and current heating. Thus, the PMMA process leads to two kinds of n-dopants, one, probably the solvents, being removed by moderate heating. On the basis of our results, we explain the n-doping of graphene partially observed after lithography $\sqrt{13 \mid 17\lfloor 19}$ by residues of PMMA. Thereby, we resolve the puzzle that 

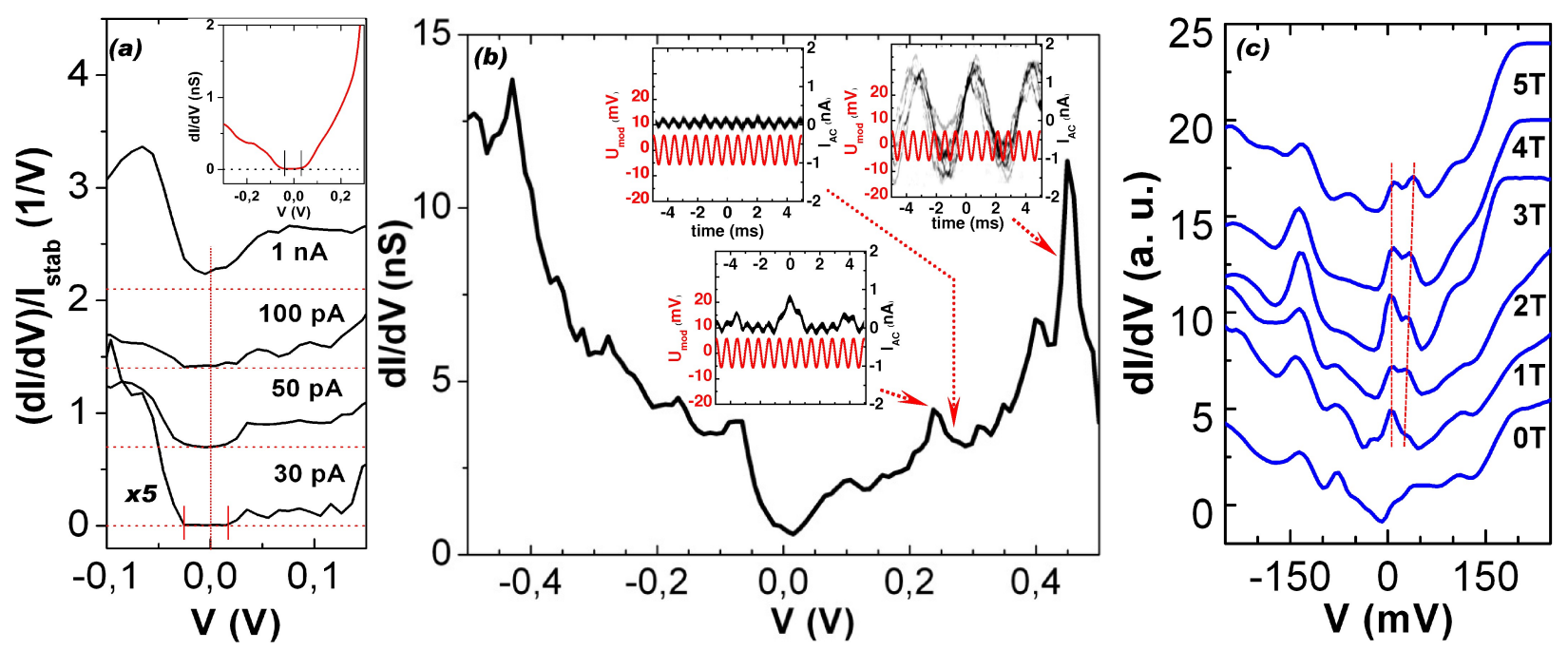

FIG. 3. (Color online) (a) $d I / d V$ spectra (normalized by stabilization current $I_{\text {stab }}$ ) taken at the same position with the same tip for different $I_{\text {stab }}$ as marked $\left(\mathrm{T}=5 \mathrm{~K}, \mathrm{~V}_{\text {stab }}=0.5 \mathrm{~V}, \mathrm{~V}_{\text {mod }}=4 \mathrm{mV}\right)$; curves are vertically displaced for clarity; dotted, horizontal lines mark $d I / d V / I_{\text {stab }}=0 / \mathrm{V}$. Inset: $\mathrm{dI} / \mathrm{dV}$ spectrum of lithographically contacted graphene $\left(\mathrm{T}=5 \mathrm{~K}, \mathrm{I}_{\text {stab }}=2 \mathrm{nA}, \mathrm{V}_{\text {stab }}=0.7 \mathrm{~V}\right.$, $\mathrm{V}_{\text {mod }}=1 \mathrm{mV}$ ); vertical lines mark the phonon gap. (b) $\mathrm{dI} / \mathrm{dV}$ spectrum taken on microsoldered graphene at higher $I_{\text {stab }}\left(\mathrm{T}=5 \mathrm{~K}, \mathrm{I}_{\text {stab }}=2 \mathrm{nA}, \mathrm{V}_{\text {stab }}=0.5 \mathrm{~V}, \mathrm{~V}_{\text {mod }}=4 \mathrm{mV}\right)$; insets show current response $\mathrm{I}_{A C}$ (black) to the oscillating tip voltage $U_{\text {mod }}$ (red) at the DC voltages marked by arrows. (c) $B$-field dependence of $d I / d V$ spectra $\left(\mathrm{T}=5 \mathrm{~K}, \mathrm{I}_{\text {stab }}=2 \mathrm{nA}, \mathrm{V}_{\text {stab }}=0.07 \mathrm{~V}, \mathrm{~V}_{\text {mod }}=10 \mathrm{mV}\right)$; curves are vertically displaced for clarity; dashed lines mark the splitting of the 0 . LL.

the known p-doping by $\mathrm{O}_{2}, \mathrm{H}_{2} \mathrm{O}^{20} \sqrt{22}$ or by Au-contacts $\frac{23 \mid 24}{2}$ cannot explain the occasionally observed negative Dirac point positions. Notice, that PMMA has recently also been found to bury p-dopants, which then cannot be removed by additional heating 13 .

Finally, we discuss STS results from the microsoldered sample. Fig. 3(a) shows a series of $\mathrm{dI} / \mathrm{dV}$ spectra recorded with the same microtip at the same position. At low current, a gap of $\Delta V \simeq 40 \mathrm{mV}$ is visible, being very similar to the gap found by Zhang et al.11. The gap was interpreted as a phonon gap, i.e. a phonon with large wave vector is required in order to tunnel into or out of K-point states. Such a gap has not been found by other groups 1012225 and we also found it only occasionally using lithographic samples (inset of Fig. 3(a)). Figure 3(a) demonstrates that the gap disappears at larger current. It reappears after reducing the current again (not shown). The onset current for closing the gap obviously depends on the microtip. This straightforwardly explains the discrepancy found by different groups. 
We believe that a local heating of the sample by the tunneling current produces enough phonons, so that the phonon annihilation can provide the required wave vector towards Kpoint states. Fig. 3(b) shows a larger scale $d I / d V$ curve ( high $I_{\text {stab }}$ ). It exhibits a number of peaks. The peaks are not related to the density of states as can be deduced from the current response to the applied modulation voltage shown as insets. At the peaks, the frequency of the response differs strongly from the excitation frequency. Since this effect is reproducible on the same position, appears at different voltages for different positions and has never been observed on $\mathrm{Au}(111)$ with the same setup, we attribute it to the properties of the graphene sample. We suggest that dielectric forces are responsible, which might lead to a non-linear mechanical movement of the flake ${ }^{26}$. Thus, $\mathrm{dI} / \mathrm{dV}$ spectroscopy on graphene seems to be very susceptible to signals not related to the LDOS.

Fig. 3(c) shows a B-field run of spectra, which does not feature series of Landau levels, although we have measured Landau and spin levels on $\operatorname{InSb}(110)$ with the same STM 27228 . Only around $0 \mathrm{~V}$, there is a peak, which develops into a doublet at higher $B$. It looks similar to the 0 . Landau level observed by STS of flat graphene either on $\mathrm{HOPG}^{25}$ or on the C-face of $\mathrm{SiC}(0001)^{29}$. The splitting of the doublet of $25 \mathrm{meV}$ at $\mathrm{B}=4 \mathrm{~T}$ corresponds exactly to the splitting values found in $\frac{25}{25}$ and

In summary, we used microsoldering in order to show that the mobility, the hysteresis and the rippling of graphene on $\mathrm{SiO}_{2}$ are barely influenced by lithography, but that PMMA and solvents lead to significant n-type doping. Moreover, we confirm a considerable improvement of cleanliness by microsoldering, which is a big advantage for future STM experiments. First STS results show that $d I / d V$ curves are strongly susceptible to effects not related to the LDOS probably due to local heating and mechanical vibrations. Nevertheless, B-field data reveal a peak which we relate to the split 0 . Landau level.

We appreciate helpful discussions with C. Stampfer and acknowledge financial support by the DFG (Mo-858/8-1 and Mo 858/11-1) and the excellence initiative of the German federal and state government.

\section{REFERENCES}

${ }^{1}$ K. S. Novoselov, A. K. Geim, S. V. Morozov, D. Jiang, Y. Zhang, S. V. Dubonos, I. V. Grigorieva, and A. A. Firsov, Science 306, 666 (2004). 
${ }^{2}$ Y. Zhang, J. W. Tan, H. L. Stormer, and P. Kim, Nature 438, 201 (2005).

${ }^{3}$ A. H. C. Neto, F. Guinea, N. M. R. Peres, K. S. Novoselov, and A. K. Geim, Rev. Mod. Phys. 81, 109 (2009).

${ }^{4}$ A. K. Geim and K. S. Novoselov, Nature Mat. 6, 183 (2007).

${ }^{5}$ J. C. Meyer, A. K. Geim, M. I. Katsnelson, K. S. Novoselov, T. J. Booth, and S. Roth, Nature 446, 60 (2007).

${ }^{6}$ V. Geringer, M. Liebmann, T. Echtermeyer, S. Runte, M. Schmidt, R. Rückamp, M. C. Lemme, and M. Morgenstern, Phys. Rev. Lett. 102, 76102 (2009).

${ }^{7}$ M. I. Katsnelson and A. K. Geim, Phil. Trans. R. Soc. A 366, 195 (2008).

${ }^{8}$ E. H. Hwang, S. Adam, and S. D. Sarma, Phys. Rev. Lett. 98, 186806 (2007).

${ }^{9}$ Ç. Ö. Girit and A. Zettl, Appl. Phys. Lett. 91, 193512 (2007).

${ }^{10}$ L. Tapasztó, G. Dobrik, P. Nemes-Incze, G. Vertesy, P. Lambin, and L. P. Biró, Phys. Rev. B 78, 2334071 (2008).

${ }^{11}$ Y. Zhang, V. W. Brar, F. Wang, C. Girit, Y. Yayon, M. Panlasigui, A. Zettl, and M. F. Crommie, Nature Phys. 4, 627 (2008).

${ }^{12}$ A. Deshpande, W. Bao, F. Miao, C. N. Lau, and B. J. LeRoy, Phys. Rev. B 79, 205411 (2009).

${ }^{13}$ T. Lohmann, K. von Klitzing, and J. H. Smet, Nano Lett. 9, 1973 (2009).

${ }^{14}$ W. Kim, A. Javey, O. Vermesh, Q. Wang, Y. Li, and H. Dai, Nano Lett. 3, 193 (2003).

${ }^{15}$ X. Wang, X. Li, L. Zhang, Y. Yoon, P. K. Weber, H. Wang, J. Guo, and H. Dai, Science 324, 768 (2009).

${ }^{16}$ J. Moser, A. Barreiro, and A. Bachtold, Appl. Phys. Lett. 91, 163513 (2007).

${ }^{17}$ M. C. Lemme, T. J. Echtermeyer, M. Baus, and H. Kurz, IEEE Elect. Dev. Let. 28, 282 (2007).

${ }^{18}$ J.-H. Chen, C. Jang, S. Adam, M. S. Fuhrer, E. D. Williams, and M. Ishigami, Nature Phys. 4, 377 (2008).

${ }^{19}$ Y.-W. Tan, Y. Zhang, K. Bolotin, Y. Zhao, S. Adam, E. H. Hwang, S. D. Sarma, H. L. Stormer, and P. Kim, Phys. Rev. Lett. 99, 246803 (2007).

${ }^{20}$ F. Schedin, A. K. Geim, E. W. Hill, P. Blake, M. I. Katsnelson, and K. S. Novoselov, Nature Mat. 6, 652 (2007).

${ }^{21}$ O. Leenaerts, B. Partoens, and F. M. Peeters, Phys. Rev. B 77, 125416 (2008).

${ }^{22}$ T. O. Wehling, A. I. Lichtenstein, and M. I. Katsnelson, Appl. Phys. Lett. 93, 202110 
(2008).

${ }^{23}$ G. Giovannetti, P. A. Khomyakov, G. Brocks, V. M. Karpan, J. van den Brink, and P. J. Kelly, Phys. Rev. Lett. 101, 26803 (2008).

${ }^{24}$ E. J. H. Lee, K. Balasubramanian, R. T. Weitz, M. Burghard, and K. Kern, Nature Nanotechnol. 3, 486 (2008).

${ }^{25}$ A. Luican, G. Li, and E. Y. Andrei, Solid State Commun. 149, 1151 (2009).

${ }^{26}$ T. Mashoff, M. Pratzer, V. Geringer, T. J. Echtermeyer, M. C. Lemme, M. Liebmann, and M. Morgenstern, arXiv:0909.0695v1 (2009).

${ }^{27}$ T. Mashoff, M. Pratzer, and M. Morgenstern, Rev. Sci. Instrum. 80, 53702 (2009).

${ }^{28}$ S. Becker, M. Liebmann, and M. Morgenstern, Phys. Rev. B (to be published).

${ }^{29}$ D. L. Miller, K. D. Kubista, G. M. Rutter, M. Ruan, W. A. de Heer, P. N. First, and J. A. Stroscio, Science 324, 1171810 (2009). 